
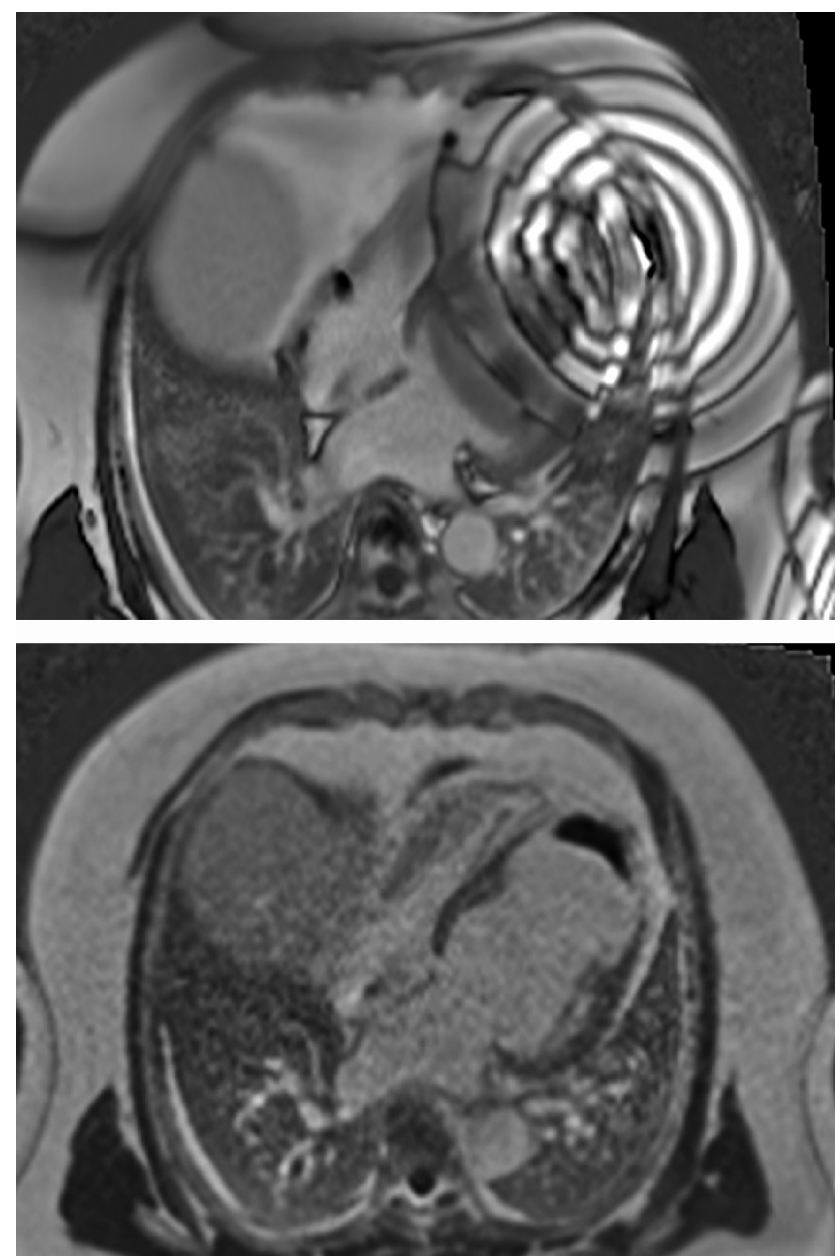

Abstract 014 Figure 1 Conventional LGE (le4) with device ar-fact and WB MOCO LGE revealing a large apical thrombus secondary to a large LAD infarct (right).

Overall, CMR with WB-MOCO LGE changed management in an additional 26 (39\%) patients compared to CMR with conventional LGE (33 patients, 49\%). This benefit was in $77 \%$ of defibrillators, $25 \%$ of pacemakers and $5 \%$ of the ILR groups.

Conclusion The WB-MOCO sequence permits high quality LGE imaging in device patients, and is robust enough for a clinical setting. This adds clinical utility particularly in defibrillator patients, also pacemaker patients but with limited utility in ILRs. Our electrophysiology department has now incorporated this into their protocol prior to ventricular tachycardia ablation.

\section{CLINICAL UTILITY OF T1 MAPPING IN CARDIAC ATTR AMYLOIDOSIS - DIAGNOSTIC PERFORMANCE AND PROGNOSTIC CAPABILITY} ${ }^{1,2} \mathrm{~K}$ Norrington, ${ }^{1} \mathrm{~A}$ Martinez-Naharro, ${ }^{1} \mathrm{~T}$ Kotecha, ${ }^{1} \mathrm{R}$ Francis, ${ }^{1} \mathrm{DF}$ Hutt, ${ }^{1} \mathrm{~T}$ Rezk, ${ }^{1} \mathrm{C}$ Quarta,
${ }^{3} \mathrm{TA}$ Treibel, ${ }^{1} \mathrm{CJ}$ Whelan, ${ }^{1} \mathrm{D}$ Knight, ${ }^{4} \mathrm{P}$ Kellman, ${ }^{5} \mathrm{FL}$ Ruberg, ${ }^{1} \mathrm{JD}$ Gillmore, ${ }^{2,3} \mathrm{~J} \mathrm{C}$ Moon,
${ }^{1} \mathrm{PN}$ Hawkins, ${ }^{1} \mathrm{M}$ Fontana. ${ }^{1}$ National Amyloidosis Centre, University College London, Royal
Free Hospital, London, UK; ${ }^{2}$ Institute of Cardiovascular Science, University College London,
London, UK; ${ }^{3}$ Barts Heart Centre, West Smithfield, London, UK; ${ }^{4}$ National Heart, Lung and
Blood Institute, National Institutes of Health, Bethesda, Maryland, USA; ${ }^{5}$ Amyloidosis Centre
and Section of Cardiovascular Medicine, Department of Medicine, Boston University, School
of Medicine, Boston Medical Centre, UK

\subsection{6/heartjn|-2017-311399.15}

Objectives Cardiac failure caused by transthyretin amyloidosis (ATTR) is an underdiagnosed clinical entity which has an important overlapping clinical phenotype with hypertrophic cardiomyopathy (HCM). Native myocardial T1 mapping by CMR is useful for diagnosis in cardiac amyloidosis. Here, we investigate the diagnostic and prognostic value of T1 mapping in the largest ATTR population studied so far as well as patients with HCM. We aimed to: 1) assess the ability of native $\mathrm{T} 1$ to diagnose cardiac amyloidosis; 2) compare native T1 to extracellular volume (ECV), and; 3) stratify prognosis.

Methods 134 wild-type ATTR (ATTRwt) (122 males, age 76 \pm 7 years), 95 mutant-type (ATTRm) (64 males, age 66 \pm 12 years) and 12 mutation carriers (4 males, age $46 \pm 8$ years) were compared to $44 \mathrm{HCM}$ patients. All subjects underwent CMR with standard SSFP-cine imaging, T1 mapping and ECV measurement. ATTR patients underwent ${ }^{99 \mathrm{~m}}$ Tc-DPD scintigraphy, the current diagnostic imaging reference standard for ATTR, with uptake determined by semi-quantitative score.

Results Native T1 and ECV were elevated in ATTR compared to HCM $(\mathrm{p}<0.001)$ (mean T1: in ATTRwt 1091 $\pm 52 \mathrm{~ms}$, in ATTRm $1084 \pm 68 \mathrm{~ms}$, in HCM $1026 \pm 64 \mathrm{~ms}$; mean ECV: in ATTRwt 0.6 \pm 0.1 , in ATTRm $0.58 \pm 0.2 \mathrm{~ms}$, in HCM 0.38 $\pm 0.1 \mathrm{~ms})$. No significant difference between native $\mathrm{T} 1$ and ECV was found between ATTRwt and ATTRm. Native T1 and ECV diagnostic performance was similar for ATTRwt and ATTRm (vs HCM: T1 AUC 0.89; ECV AUC 0.93; p=0.11
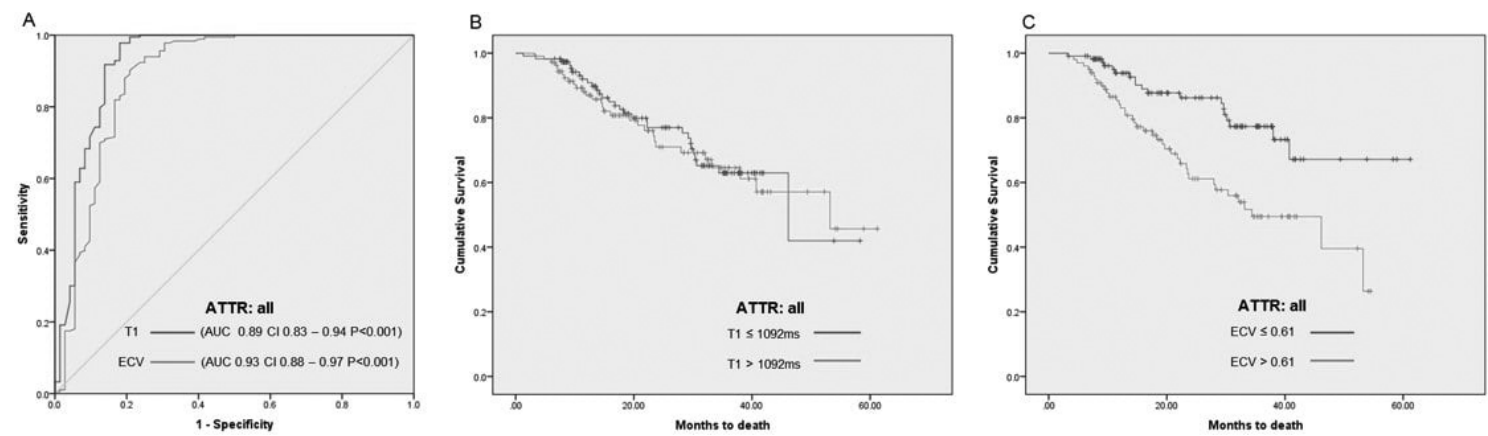

Abstract 015 Figure 1 Diagnostic performance and prognostic capability. (A) Receiver operator characteristics curve (ROC) for the discrimination of possible or definite transthyretin (ATTR) cardiac amyloidosis by native T1 and ECV from HCM. Kaplan-Meier survival curves for (B) pre-contrast myocardial T1 and (C) extracellular volume at bolus. The median native myocardial T1 (1092ms) (B) and median extracellular volume (ECV) (0.61) (C) were used as the respective cut points for survival. 
Cine
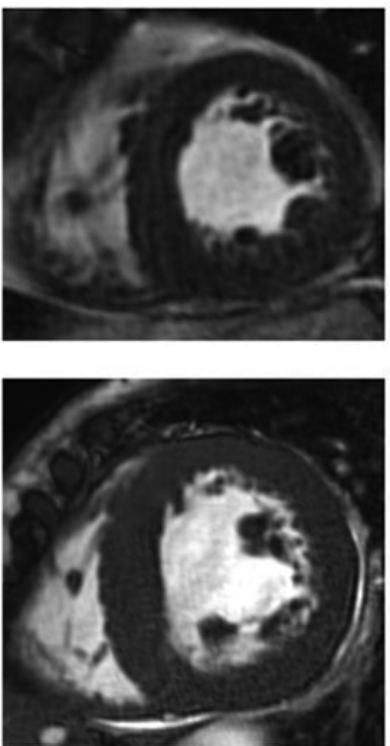

T1 Map
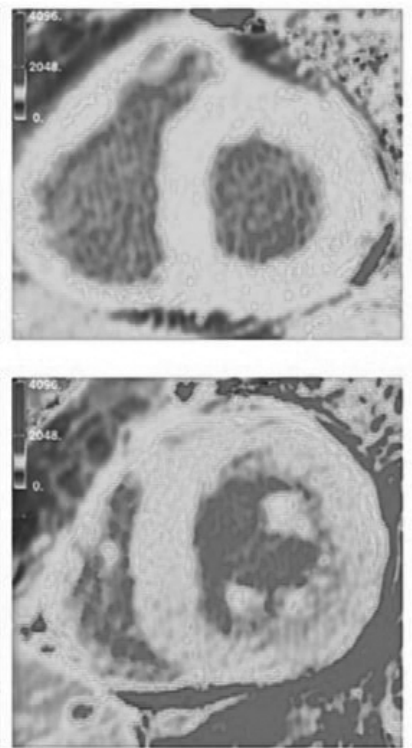

LGE
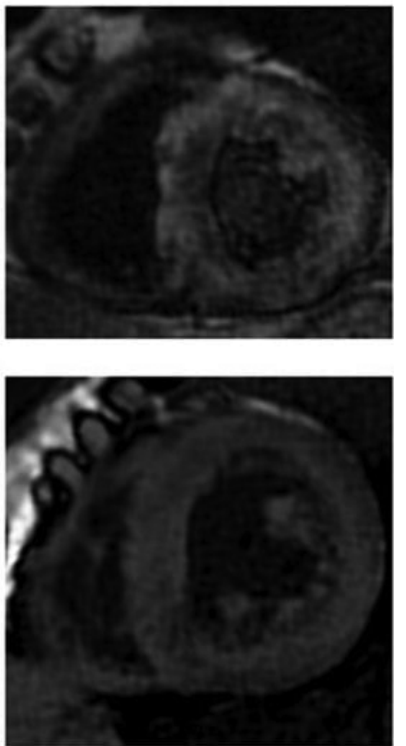

Abstract 015 Figure 2 Characteristics Examples from CMR Scans. CMR end-diastolic cine (far left), shortened modified look locker inversion recovery native $\mathrm{T1}$ map (middle) and late gadolinium enhancement (LGE) images (far right), in two patients with definite transthyretin amyloidosis (ATTR amyloidosis), one (top) with normal T1 map, mildly eleveted native myocardial T1 (1039ms) and very elevated ECV (0.7), the other (bottom) with abnormal T1 map, markedly elevated native myocardial T1 (1224ms) and very elevated ECV (0.7).

for the significance of the difference between areas under the ROC curves). During follow-up, 63 deaths occurred: 34 ATTRwt, 29 ATTRm. Whilst native T1 was not predictive of death, ECV was (HR, 1.130; 95\% confidence interval, 1.06$1.2 ; \mathrm{p}<0.001)$ and remained independent after adjustment for age, N-terminal pro b-type natriuretic peptide, left ventricular (LV) ejection fraction, E/E', LV mass index, global longitudinal strain and tricuspid annular plane systolic excursion.

Conclusions CMR-determined native myocardial T1 and ECV provide excellent diagnostic accuracy for identification of ATTR cardiac amyloidosis and both variables track DPD-determined amyloid burden well. In this study, whilst T1 was not a predictor of mortality, ECV was independently associated with mortality.

\section{USPIO-ENHANCED CMR COMPREHENSIVE METHODOLOGICAL INVESTIGATION AND APPLICATION IN ACUTE MI}

1,2Jakub Lagan, ${ }^{1}$ David Clark, ${ }^{1}$ Anna Reid, ${ }^{1}$ Gavin Lewis, ${ }^{2}$ Josephine Naish, ${ }^{2}$ James Fildes, ${ }^{2}$ Andrew Trafford, ${ }^{2}$ William Critchley, ${ }^{3}$ Erik B Schelbert, ${ }^{1}$ Matthias Schmitt, 'Phillip Foden, ${ }^{1,2}$ Christopher A Miller. 'University Hospital of South Manchester, UK; ${ }^{2}$ University of Manchester, UK; ${ }^{3}$ University of Pittsburgh, UK

\subsection{6/heartjnl-2017-311399.16}

Introduction Quantification of active myocardial inflammation may improve diagnosis, guide management and provide trial end-points for novel therapies. Ultrasmall particles of iron oxide (USPIO) are phagocytosed by activated leukocytes and USPIO-enhanced CMR is increasingly used to assess tissue inflammation. We aimed to; 1 . Compare T2* imaging with T1 mapping, which is proposed as an alternative for 'native' cardiac iron measurement; 2. Determine whether imaging at a single time point post-USPIO is sufficient to detect active accumulation in tissue; 3. Determine whether USPIO signal from infarct and remote zones in acute myocardial infarction (MI) reflects active myocardial accumulation or passive 'washthrough' in oedematous myocardium.

Methods Four healthy volunteers and six patients with acute MI underwent 1.5T CMR, including T1 and T2* mapping, before and at multiple time points following $4 \mathrm{mg} / \mathrm{kg}$ ferumoxytol.

Results Normalised T2* of spleen, an organ with high active leukocyte activity, dropped post-USPIO and remained low over the study period (Figure 1), with no correlation seen between spleen $\mathrm{T} 2 *$ and blood T1 (rho $=-0.43, \mathrm{p}=0.875)$. In comparison, T1 recovery in spleen correlated strongly with $\mathrm{T} 1$ recovery in blood (rho=0.924, $\mathrm{p}<0.001)$. In healthy myocardium, an organ with low leukocyte activity, T1 and T2*recovery both correlated strongly with blood T1 (rho=0.953, p $<0.001$; rho $=0.935, \mathrm{p}<0.001$ respectively).

In MI, absolute T2* values dropped and remained significantly lower in infarcted (15 vs $27 \mathrm{~ms}, \mathrm{p}<0.001,22$ vs $38 \mathrm{~ms}$, $\mathrm{p}=0.001)$ and remote myocardium (21 vs $27 \mathrm{~ms}, \mathrm{p}=0.05,28$ vs $38 \mathrm{~ms}, \mathrm{p}=0.024)$ compared to healthy controls. T2* and T1 recovery curves post-USPIO were significantly different in both infarcted $(p=0.028)$ and remote myocardium $(p=0.004$; Figures 2).

Conclusions $0 \mathrm{~T} 2 *$ is sensitive to active tissue accumulation of USPIO, likely because T2* reflects field gradients, such as those generated by compartmentalised (phagocytosed) USPIO. T1, which is due to short range dipolar interactions that reduce as USPIOs wash-out, simply tracks passive wash-through. T1 is therefore less suitable for detecting active leukocytes. Measuring T2* at a single time point post-USPIO is insufficient to determine tissue accumulation from passive wash-through. USPIO signal from infarct and remote zones in acute MI appears to genuinely reflect active myocardial accumulation, presumably due to phagocytosis by activated leukocytes. 